\title{
Athletes returning to play after cervical spine or neurobrachial injury
}

\author{
Shiveindra Jeyamohan · James S. Harrop • \\ Alex Vaccaro · Ashwini D. Sharan
}

Published online: 26 August 2008

(C) Humana Press 2008

\begin{abstract}
The treatment algorithms for athletes with spine injuries follow similar guidelines as those for nonathletes in terms of deciding between surgical intervention and non-operative management. However, the athlete has unique postoperative demands and the decision to "allow" an athlete to return to competitive sports after a spinal or plexus injury can be difficult. This article reviews the several studies, available guidelines and peer-reviewed publications to aid in the decisions to allow athletes to return to sports. A set of recommendations concerning return to play after a spinal injury is provided.
\end{abstract}

Keywords Cervical - Return to sports - Spine ·

Injury $\cdot$ Neuropraxia

\section{Introduction}

Approximately $10 \%$ of the 10,000 cervical spine injuries that occur annually in the United States are the result of an athletic injury [1]. Fortunately, associated spinal cord

S. Jeyamohan

Jefferson Medical College, Philadelphia, PA 19107, USA

e-mail: Shiveindra.jeyamohan@jefferson.edu

J. S. Harrop $(\varangle) \cdot$ A. D. Sharan

Department of Neurological Surgery, Thomas Jefferson

University, Philadelphia, PA, USA

e-mail: James.harrop@jefferson.edu

A. D. Sharan

e-mail: Ashwini.sharan@jefferson.edu

A. Vaccaro

Department of Orthopaedic Surgery, Thomas Jefferson

University, Philadelphia, PA, USA

e-mail: Alexander.vaccaro@jefferson.edu injuries are infrequent. The principles of initial resuscitation, immobilization and neurological survey for general trauma can be applied to sports-injured patients [2]. Likewise, acute treatment algorithms for athletes with spine injuries follow similar guidelines as those for non-athletes, with common predicaments pertaining to deciding between surgical intervention and non-operative management.

Due to unique postoperative demands, the decision to "allow" an athlete to return to competitive sports after a spinal injury can be difficult. This decision is influenced by the presence of trauma-induced spinal deformity, the adequacy of postoperative/post-injury rehabilitation and the extent and/or location of the surgery or injury.

Morganti et al. [3], searching for a consensus amongst practitioners, analyzed the practices of members of the Herodius Sports Medicine Society and Cervical Spine Research Society. Through a questionnaire, the polling revealed neither consensus nor a correlation between experience, specialty, training, publications, or guidelines and their decisions to allow athletes to return to sports. The conclusion of this report was that no such correlation exists and that physicians' treatment patterns seemed, at best, arbitrary.

Despite this lack of agreement, there are a number of works that have been published in this area. These may help to guide practitioners' treatment of this unique patient population. The purpose of this article is to review available guidelines and peer-reviewed publications in order to distill a set of recommendations concerning return to play after a spinal injury.

\section{Clinical terminology}

Injured athletes can present to a spine physician in many settings, such as during an in-office visit or on the field 
during a competition. Furthermore, the nature of the injury presentation can vary. For example, some athletes report a sudden "pop" in the neck that may be associated with axial or appendicular pain. There may be varying gradations of altered sensations or paresthesiae. Weakness can be subjective or objective.

A wide variety of presentations exist. As such, the approach to theses problems begins with an accurate description of the injury. It is important to note whether the athlete was able to complete the athletic event or if the pain and symptoms were too great to allow continued play. An understanding of the precise mechanism of injury and subsequent immobilization maneuvers is also paramount. A complete neurological examination to detect objective abnormalities of sensation or strength is mandatory. Imaging studies can range from plain radiographs to advanced modalities such as computerized tomography (CT) or magnetic resonance imaging (MRI).

The goal of this evaluation is to formulate the most accurate diagnosis of the traumatic spinal event. This step is crucial to prognosticating the likelihood of subsequent serious spinal injury and influences the decision to return to play. These diagnoses include:

- Stingers are caused by an impact that produces posterolateral neck extension and resultant narrowing of the ipsilateral cervical neuroforamen. This posture can cause nerve-root compression or a stretch of the brachial plexus. Clinical symptoms include temporary burning or dysesthetic radiating pain down one upper extremity. This is sometimes accompanied by motor weakness as well. Isolated stingers are common amongst athletes; however, repeat stingers are more serious and can herald an event causing permanent deficit in the future. If play modification fails to settle the symptoms, withdrawal of the activity may be necessary to prevent long-term damage [1].

- Burning hands syndrome is characterized by burning dysthesias bilaterally in the upper extremities, rarely involving the lower extremities. Associated ligamentous injury or cervical fracture is common. Burning hands syndrome can be considered as a mild form of a central cord syndrome. Return to play is typically contraindicated [1].

- Spinal cord neuropraxia (SCN) is transient tetraparesis. It has a reported incidence of 1.3 per 10,000 athletic participants [4]. The proposed mechanism of injury is direct axial compression, but it can be accompanied by hyperflexion or extension. Patients present with bilateral paresthesias and variable degrees of weakness in the upper and lower extremities. The neurological symptoms usually last only $10-15 \mathrm{~min}$; however, in distinction to a spinal cord injury (SCI), they resolve by $48 \mathrm{~h}$.
- Permanent tetraparesis or tetraplegia (spinal cord injury). Though underlying cervical stenosis can be present, neurological deficit is associated with a spinal fracture or dislocation. Impact on the top of the head with the neck in forward-flexion (i.e. spear tackling) removes the protective effect of cervical lordosis; this is a commonly cited injury mechanism. Athletes with permanent neurological injury should be excluded from all contact sports [1].

\section{Radiographic evaluation}

A substantial body of literature has attempted to correlate morphological features of the cervical spine to the risk of neurological injury during sports activity. Most studies have hypothesized that athletes with smaller spinal canals might be more prone to injury.

Kang et al. [5] reported on 283 patients with cervical fractures and dislocations, specifically analyzing the space available for the spinal cord at the level of injury, the sagittal diameter of the canal at uninjured levels and the Pavlov ratio at uninjured levels. The canal measured a mean of $10.5 \mathrm{~mm}$ in patients with complete SCI, $13.1 \mathrm{~mm}$ in those with incomplete SCI, $15.9 \mathrm{~mm}$ with nerve-root injury and $16.7 \mathrm{~mm}$ for those without neurological deficit. Statistical differences were found between the groups $(P<0.001)$, supporting the notion that a larger canal diameter was somewhat protective against injury. At the uninjured level, the mean Pavlov ratio was 0.82 in those with complete SCI and 0.84 for those with incomplete SCI. In those with nerve-root injury, the Pavlov Ratio was 0.96 in patients. These differences were statistically significant $(P<0.005)$ in that the smaller Pavlov ratio correlated with a higher risk for SCI.

Torg et al. $[4,6,7]$ reported on 110 patients that experienced transient neuropraxia from a cervical injury during sports. Importantly, no patient in the study sustained permanent or catastrophic neurological injury. Similar to the Pavlov ratio, Torg described a ratio defined as anteroposterior diameter of the spinal canal/vertebral body (SC/VB) ratio. In their calculations, individuals with smaller SC/VB ratios $(0.65 \pm 0.1)$ were more likely to sustain recurrences when compared to those with higher ratios $(0.72 \pm 0.1)$. Other findings of interest included that individuals returning to football after an injury had a higher recurrence rate compared to other sports. Ultimately, Torg et al.'s recommendations were that if an athlete completely recovered without complication after one or two episodes of SCN, then he may safely return to play. Those with larger SC/VB ratios were deemed appropriate to return to play as they are least likely to suffer from recurrences. Torg et al. 
concluded that athletes with mechanically stable spines who have experienced transient paralysis are not at increased risk for catastrophic SCI compared with those who had never sustained transient paresis.

Despite its apparent utility, Torg et al. $[4,6,7]$ have not recommended using the SC/VB ratio as a screening mechanism for determining whether an athlete can participate in contact sports. In 1996, Torg et al. reported on the ratio's poor positive predictive value $(0.2 \%)$ when using the recommended criterion of 0.80 . Therefore, the Torg ratio can help stratify the risk of a neuropraxic event but it is, by itself, not a guideline for athletes return to contact sports $[4,6,7]$.

Eismont et al. reported 98 patients with spinal fracturedislocation and neurological injury after sports injuries [8]. This study differed from the prior studies in that the risk of injury from fractures was assessed. This group studied the sagittal diameter of each cervical vertebra and reported the average values in those patients with complete SCI, incomplete SCI and no deficit (Table 1). Similar to Torg, they found that an athlete with a larger canal was less likely to have a neurological deficit after a spinal fracture or dislocation.

\section{Previously reported guidelines}

Despite the apparent lack of consensus amongst practitioners and the failure to find a highly sensitive and specific parameter with which to predict those at greatest risk, several authors have elaborated guidelines concerning return to play after injury. For fear of discarding all of these for not being "evidence-based", one must consider the difficulty in accruing a sound body of evidence on this topic. The following guidelines, therefore, borrow heavily from the authors' experience, which may be the "best available" information on this topic.

Bailes et al. [9] guided return to play based on the classification of the neurological deficit. Athletes with Type 1 injuries (permanent SCI) were barred from any further participation within the contact sport. Those with Type 2 injuries (transient SCI, usually without radiological findings) were allowed to participate in contact athletic

Table 1 Eismont et al.'s table of average sagittal cervical sizes for injury

\begin{tabular}{llll}
\hline & Complete SCI $(\mathrm{mm})$ & Incomplete SCI $(\mathrm{mm})$ & No deficit $(\mathrm{mm})$ \\
\hline C2 & 18.6 & 20.0 & 22.0 \\
C3 & 17.0 & 18.0 & 19.4 \\
C4 & 16.1 & 17.3 & 18.9 \\
C5 & 16.0 & 17.3 & 18.7 \\
C6 & 16.1 & 17.1 & 18.7 \\
C7 & 16.3 & 17.5 & 18.7 \\
\hline
\end{tabular}

events as long as the injury did not recur. Athletes with Type 3 injuries (neurological deficits in addition to radiological abnormalities) need further enquiry regarding their suitability for return to athletic play. The group also suggested electromyography several weeks after the traumatic injury in order to confirm nerve involvement in injuries involving the brachial plexus.

Morganti [10] associated "transient...neurapraxia...[as] related to forced hyperflexion, hyperextension, or axial compression of the cervical spine". This perhaps illustrates why Torg's ratio has a poor predictive value, as it only represents anatomical dimensions and not functional mechanics. Furthermore, it has been reported that $50 \%$ of athletes experience brachial plexus neurapraxia at some point in their college athletic careers.

Other studies, have illustrated that a substantial percentage of athletes who have had neurological injury will

Table 2 Absolute contraindications to return to play

Previous transient quadriparesis

$>2$ previous episodes of cervical cord neurapraxia or transient quadriparesis

Evidence of cervical myelopathy based on clinical history or physical examination

Continued cervical discomfort, decreased range of motion, neurological deficit (from baseline)

Postsurgical patients

C1-C2 fusion

$\mathrm{s} / \mathrm{p}$ cervical laminectomy

$\mathrm{s} / \mathrm{p}$ anterior or posterior cervical fusion $\geq 3$ levels

Soft tissue injuries

Asymptomatic ligamentous laxity ( $>11^{\circ}$ kyphotic deformity)

C1-C2 hypermobility (Atlanto-dens interval $>3.5 \mathrm{~mm}$ in adult or $>5 \mathrm{~mm}$ in child)

Radiographic evidence of a distraction-extension injury

Symptomatic cervical disc herniation

Other radiographic findings

Plain films

Presence of spear-tackler's spine

Multi-level Klippel-Feil anomaly

Healed subaxial spine fracture with evidence of sagittal or coronal plane deformity

Evidence of ankylosing spondylitis or diffuse idiopathic skeletal hyperostosis

Evidence of rheumatoid arthritis

MRI

Basilar invagination

Residual cord encroachment following healed, stable sub-axial spine fracture

Presence of cervical spinal cord abnormality CT

Fixed atlanto-axial (C1-C2) rotatory subluxation

Occipital-C1 assimilation 
Table 3 Relative contraindications to return to play

Prolonged symptomatic burner/stinger or transient quadriparesis $>24 \mathrm{~h}$

$\geq 3$ prior episodes of stinger/burner; patient must have full return to baseline cervical range of motion and neurological status without an increase in baseline neck discomfort

$\mathrm{s} / \mathrm{p}$ healed two level anterior or posterior fusion surgery \pm instrumentation

Table 4 No contraindications to return to play

Fractures
Healed $\mathrm{C} 1$ or $\mathrm{C} 2$ fracture with normal cervical range of motion
Healed subaxial fracture without sagittal plane deformity
Asymptomatic clay shoveller's ( $\mathrm{C} 7$ spinous process) fracture
Congenital
Single-level Klippel-Feil anomaly not involving the C0-C1
articulation
Spina bifida occulta
Torg ratio $<0.8$ and asymptomatic
Degenerative/Postsurgical
Cervical disc disease treated successfully with only occasional
neck stiffness or pain with no evidence of a neurological deficit
s/p healed single-level anterior or posterior cervical
fusion \pm instrumentation
s/p single or multi-level posterior cervical laminoforaminotomy
Other
$<3$ previous episodes of burner/stinger lasting $<24 \mathrm{~h}$, with full
cervical range of motion and no evidence of a neurological
deficit
1 episode of transient quadriparesis with full cervical range of
motion, no evidence of neurological deficit and no evidence
of herniated disc or radiographic instability

go on to experience further events throughout their careers [10]. However, it was later found that players returning after full recovery without radiological findings were at no increased risk when compared to asymptomatic athletes in the same sport. In a recent review, Vaccaro et al. [11] synthesized the existing literature to delineate a list of absolute and relative contraindications for return to play (Tables 2-5).

\section{Special circumstances}

Spear-tackler's spine

A spear-tackler's spine is defined by developmental narrowing of the cervical canal, persistent straightening or reversal of the normal cervical lordosis, simultaneous preexistent post-traumatic radiographic abnormalities of the cervical spine, and documented use of spear-tackling techniques in football. These attributes place the spinal
Table 5 Guidelines for return to play after successful surgery

\begin{tabular}{ll}
\hline Status & Surgery \\
\hline Safe to return & Normal ROM of C-spine after healing \\
& C1 or C2 fractures \\
& Healed subaxial fractures lacking \\
& sagittal plane deformity \\
& Asymptomatic C7 spinous process \\
& fracture (clay shoveller's fracture) \\
Further enquiry; certain & Two level surgical fusion, not including \\
contraindications exist & posterior segmental lateral mass \\
& screw fixation \\
Absolute exclusion from & C1-C2 surgical fusion \\
further athletic play & Cervical laminectomy \\
& Three level anterior or posterior surgical \\
& fusion \\
& Radiographic evidence of segmental \\
& instability \\
& Radiographic evidence of C1-C2 \\
& instability \\
& Radiographic evidence of distraction/ \\
& extension cervical spine injury \\
& Healed subaxial spine fracture with \\
& sagittal plane kyphosis or coronal \\
& plane deformity \\
\hline &
\end{tabular}

cord at risk during axial loads with head-impact playing. Having a spear-tackler's spine is an absolute contraindication to return to athletic activity because of its association with severe injury.

Previous cervical spine fusion

Kim et al. [1] polled 450 members of the North American Spine Society about their preferences concerning returning to sports after a successful spinal fusion. Of those that responded, 57\% discouraged return to play in some sports. Nevertheless, $80 \%$ reported that they have treated athletes who successfully returned to sports after fusion, $62 \%$ of whom played college sports and $18 \%$ of whom were professional. Importantly, these statistics did not include football players. About $90 \%$ reported that they would wait a year to ensure that a cervical fusion was solid and mature and confirm that the patient had full range of motion. About $15 \%$ reported that they would be more likely to utilize rigid instrumentation in athletes than in non-athletes. Tables 2-5 summarize these groups's recommendations regarding return to play after fusion.

\section{Conclusion}

There is some prior literature establishing recommendations on return to play in athletes having sustained a 
cervical injury secondary to trauma. These reports provide, at best Class III evidence. Thus, firm conclusions cannot be made and treatment recommendations must be made on individual bases rooted in good clinical judgement. The literature suggests that $\mathrm{SCN}$ in a patient with a larger $\mathrm{SC} /$ VB ratio and no mechanical instability should have full return of function at no significant future risk for catastrophic injury. However, once a SCI has been sustained, return to sports is not advised. Following successful spinal fusion and a complete recovery, return to sports may be an option in some situations.

\section{References}

1. Kim D, Vaccaro A, Berta S. Return to play criteria in athletes with traumatic injuries to the cervical spine. Curr Opin Orthop. 2003;14:170-3.

2. American College of Surgeons. ATLS, Advanced Trauma Life Support Program for Doctors. American College of Surgeons, 2004.
3. Morganti C, Coleen SA, Albanese SA, et al. Return to play after cervical spine injury. Spine. 2001;26:1131-6.

4. Torg JS, Guille JT, Jaffe S. Injuries to the cervical spine in American football players. J Bone Joint Surg. 2002;84A:112-22.

5. Kang JD, Figgie MP, Bohlman HH. Sagittal measurements of the cervical spine in subaxial fractures and disloactions. J Bone Joint Surg. 1994;76A:1617-28.

6. Torg JS, Corcoran TA, Thibault D, et al. Cervical cord neurapraxia: classification, pathomechanics, morbidity, and management guidelines. J Neurosurg. 1997;87:843-50.

7. Torg JS, Pavlov H, Genuaria SE, et al. Neurapraxia of the cervical spinal cord with transient quadriplegia. Patient Care Manag. 1986;68:1354-70.

8. Eismont FJ, Clifford S, Goldberg M, Green B. Cervical sagittal spinal canal size in spine injury. Spine. 1994;9:663-6.

9. Bailes JE, Hadley MN, Quigley MR, et al. Management of athletic injuries of the cervical spine and spinal cord. Neurosurgery. 1991;29:491-7.

10. Morganti C. Recommendations for return to sports following cervical spine injuries. Sports Med. 2003;33:563-73.

11. Vaccaro A, Harrop J, Daffner S, et al. Acute cervical spine injuries in the athlete: diagnosis, management, and return-to-play. Int SportMed J. 2003;4(1):1-5. 